\title{
CONVERGENCE GROUPS ARE FUCHSIAN GROUPS
}

\author{
DAVID GABAI
}

\begin{abstract}
A group of homeomorphisms of the circle satisfying the "convergence property" is shown to be the restriction of a discrete group of Mobius transformations of the unit disk. This completes the proof of the Seifert fiber space conjecture and gives a new proof of the Nielson realization problem.
\end{abstract}

A Fuchsian group $F$ is a discrete subgroup of the group of Mobius transformations on the unit disc $D^{2}$ in $R^{2}$.F restricts to a subgroup $G$ of $\operatorname{Homeo}\left(S^{1}\right)$ which satisfies the following convergence property [GM]. Given a sequence of distinct elements of $G$, then there exists $x, y \in S^{1}$ and a subsequence $\left\{f_{i}\right\}$ such that on $S^{1}-\{x, y\} \quad f_{i} \rightarrow y, f_{i}^{-1} \rightarrow x$ uniformly on compact sets. A group $G \subset \operatorname{Homeo}\left(S^{1}\right)$ with this property is called a convergence group. We announce the following result. The details can be found in $[\mathrm{G}]$.

Theorem 1. $G$ is a convergence group if and only if $G$ is conjugate in $\operatorname{Homeo}\left(S^{1}\right)$ to the restriction of a Fuchsian group.*

A Seifert fibred space is a compact 3-manifold $M$ which is almost an $S^{1}$ bundle over a compact surface, i.e. there exists a projection $\pi: M \rightarrow N$ such that for each $x \in N$ there exists a $D^{2}$ neighborhood of $x$ such that $\pi^{-1}\left(D^{2}\right)=D^{2} \times S^{1}$ and $\pi\left(\left(r, \theta_{1}\right),\left(1, \theta_{2}\right)\right)=\left(r, p \theta_{1}+q \theta_{2}\right)$ where $p \neq 0$ and $p, q$ are relatively prime and depend on $x$ and $\theta \in \mathbb{R} \bmod 2 \pi$.

Corollary 2 (Seifert Fibred Space Conjecture). Let $M$ be a compact, orientable, irreducible (i.e. every smooth embedded $S^{2}$ bounds a 3-cell) 3-manifold with infinite $\pi_{1}$, then $M$ is a Seifert fibred space if and only if $\pi_{1}(M)$ contains a cyclic normal subgroup.

Received by the editors January 7, 1991 and, in revised form, April 20, 1991.

1980 Mathematics Subject Classification (1985 Revision). Primary 57S25; Secondary $20 \mathrm{H} 10,57 \mathrm{~N} 05,57 \mathrm{~N} 10$.

Partially supported by NSF Grant DMS-8902343 and a Sloan Foundation Fellowship.

*Andrew Casson has also announced, using different methods, a proof of Theorem 1. 
Idea of Proof. Mess [M] reduced the Seifert fibred space conjecture as follows to showing that convergence groups are isomorphic to Fuchsian groups. If $J$ is a cyclic normal subgroup and $M$ is closed, then he first shows that the covering space of $M$ with fundamental group $J$ is $\stackrel{\circ}{D}^{2} \times S^{1}$. The action of $G=\pi_{1}(M) / J$ on $\stackrel{\circ}{D}^{2} \times S^{1}$ via covering transformations, descends to a vaguely defined action on $\stackrel{\circ}{D}^{2}$. It turns out that the action is (in Mess' words) coarse quasi-isometric to either the Euclidean plane or the hyperbolic plane. In the first case $G$ is isomorphic to the fundamental group of an Euclidean orbifold. In the latter case $G$ induces a well-defined convergence group action on the circle at infinity. Theorem 1 implies that $G$ is isomorphic to a Fuchsian group, which is the fundamental group of a hyperbolic orbifold. Thus $\pi_{1}(M)$ is isomorphic to the fundamental group of a Seifert fibred space $N . M$ and $N$ are closed and aspherical, since they are both covered by $\mathbb{R}^{3}$, thus they are homotopy equivalent. By Scott [S2] they are homeomorphic.

The Seifert fibred space conjecture was established for Haken manifolds in [JS] using [W1] and [GH].

The Thurston Geometrization conjecture [Th] asserts that the sphere [Kn, Mi] and torus decompositions [JS, J] cut an orientable 3-manifold into geometric pieces. Thurston's monster theorem is the proof of this conjecture in the case that $M$ is Haken. The unresolved cases were of three types: $\pi_{1}(M)$ is finite, $\pi_{1}(M)$ contains an infinite cyclic normal subgroup, and $\pi_{1}(M)$ is infinite and does not contain an infinite cyclic normal subgroup. Manifolds of the first type are conjecturally quotients of $S^{3}$ via orthogonal actions (this includes the Poincare Conjecture). The hyperbolization conjecture is that manifolds of the third type are hyperbolic manifolds. The Seifert fibred space conjecture was that manifolds of the second type were Seifert fibred spaces.

Corollary 3 (Torus Theorem). If $M$ is an orientable, irreducible 3-manifold and $Z \oplus Z \subset \pi_{1}\left(M^{3}\right)$, then $M$ is either Seifert fibred or contains an incompressible torus (i.e. an embedded torus whose induced $\pi_{1}$ map is injective).

Proof. Waldhausen [W2] announced the (classical) torus theorem, i.e., if $M$ is Haken and $Z \oplus Z \subset \pi_{1}(M)$, then $M$ is Seifert fibred or contains an incompressible torus. Feustel [F1, F2] wrote the first proofs. The torus theorem was generalized to the strong torus 
theorem of Scott [S1] which asserts that if $Z \oplus Z \subset \pi_{1}(M)$, then either $M$ contains an incompressible torus or $\pi_{1}(M)$ contains a cyclic normal subgroup. If $M$ is not compact, then it is easy to find an incompressible torus. Corollary 2 deals with the remaining case.

The following result is an immediate consequence of Corollary 2 and [E].

Corollary 4. A compact, oriented, irreducible 3-manifold $M$ with infinite $\pi_{1}$ has a $C^{n} \quad 1 \leq n \leq \infty$ foliation by circles if and only if $\pi_{1}(M)$ has a cyclic normal subgroup.

Corollary 5 (Nielsen Realization Problem). If $\chi(S)<0, S$ a connected closed surface, then every finite subgroup $F$ of $\pi_{0}$ Homeo $(S)$, (the group of isotopy classes of homeomorphisms of $S$ ) can be realized as a group of isometries of a hyperbolic structure on $S$.

In the celebrated paper [K], Kerckhoff obtained a positive solution to Nielsen's problem. He showed that $F$ fixed a unique point of Teichmuller space, hence $F$ acts on $S$ as a group of isometries with respect to the hyperbolic metric corresponding to that point. See Wolpert [W] for another Teichmuller theory proof and [Z] for a history of the problem through 1980.

Our proof is the one Nielsen [N] had in mind in 1942. He observed that given any hyperbolic metric on $S, \pi_{1}(S)$ induces a Fuchsian action on the hyperbolic plane via covering transformations, hence an action on $S^{1}$. The lifts of two isotopic homeomorphisms to hyperbolic space induce the same map on $S^{1}$. Thus $F$ induces a group $G$ of $\operatorname{Homeo}\left(S^{1}\right)$ which has $\pi_{1}(S)$ as a normal subgroup of finite index (and hence $G$ is a convergence group). He observed that the realization problem is solved if $G$ is conjugate to a Fuchsian group.

Nielsen showed that if $F$ is cyclic and $G$ contains a simple hyperbolic element, (the convergence group analogue of a simple closed curve in a surface), then $G$ is conjugate to a Fuchsian group. Following Nielsen, Zieschang $[\mathrm{Z}]$ and Tukia $[\mathrm{T}]$ reduced the realization problem and more generally the convergence group problem to the following statement, which is what we establish.

If $G$ is a nonelementary, orientation-preserving convergence group which has no simple hyperbolic elements, then there exists an extension of $G$ to a $D^{2}$-convergence group whose restriction to $\stackrel{\circ}{D}^{2}$ is properly discontinuous. 
Each element of a convergence group has either 0,1 , or 2 fixed points and is conjugate to a rotation of finite order, a parabolic element, or a hyperbolic element, i.e., a function $h$ with exactly two fixed points such that in the neighborhood of one $h$ is expanding and in a neighborhood of the other $h$ is contracting.

We now explain the key idea. Given a hyperbolic element $h$, draw in $D^{2}$ a properly embedded path (called an axis) from one fixed point to the other. Now draw axes for all the elements conjugate to $h$. Let $X$ be the union of these axes. Several of these may appear as in Figure 1(a). $h$ is a simple hyperbolic element if none of these axes cross, as in Figure 1(b). The advantage of a simple element is that there is a natural extension of $G \mid S^{1}$ to $S^{1} \cup X$. This is one of the main observations in Nielsen, Zieschang, and Tukia's work. One then extends the action to the complementary pieces. It is the moral equivalent of chopping up a surface along simple curves to obtain a disc.

In our approach, the nonsimple axes are highly advantageous. The idea being that if one could find a conjugacy class $[h]$ such that all the axes of the conjugates chop up $\stackrel{\circ}{D}^{2}$ into compact discs and the combinatorial configuration of lines was invariant under the group, then one immediately obtains an extension of $G$ to $D^{2}$. The problem with this approach, indeed the main difficulty of the entire problem, is how to configure a triple of distinct axes which intersect each other nontrivially as in Figure 2.

Elliptic elements, which were the nemesis of the other approaches are enlisted to serve as decision makers. In hyperbolic geometry,

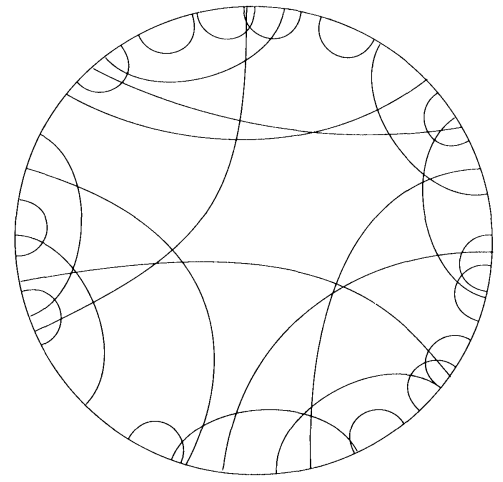

(a)

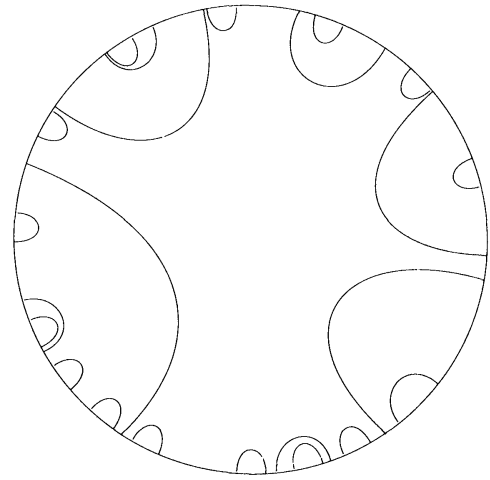

(b)

Figure 1 

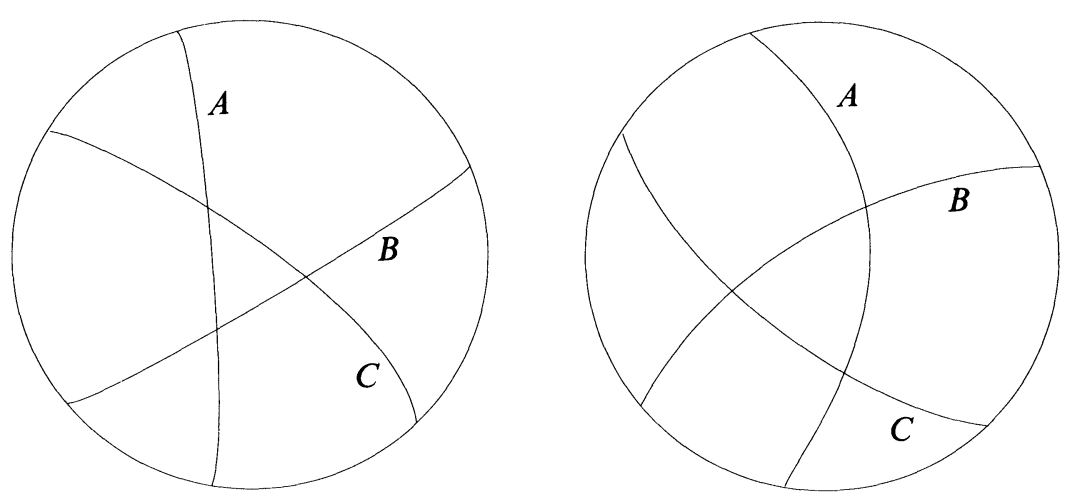

The Heart of the Problem

How to Configure $A, B$, and $C$ ?

FIGURE 2

if $A$ is an oriented axis for a hyperbolic element $h$ (oriented from the expanding fixed point $N_{A}$ to the contracting fixed point $P_{A}$ ) and $\alpha$ is an elliptic element of order 2 with fixed point $a$, then we can determine whether $a$ (resp. $\alpha$ ) is to the left, right, or on $A$ (resp. $h$ ) by simply asking whether $\alpha\left(N_{A}\right)$ is to the left, right, or on A, i.e. by inspecting the action of $h$ and $\alpha$ on $S^{1}$. In an analogous way if $\alpha$ is an elliptic element of order 2 and $h$ is a hyperbolic element of a convergence group, we can decide whether $\alpha$ is to the left, right, or on $h$. Thus an elliptic element would configure a triple as in Figure 3 on p. 400.

The proof of Theorem 1 involves two steps.

Step 1. There exists a good hyperbolic, elliptic pair $(f, \alpha)$, i.e. for each $\alpha \in[\alpha]$ and $f \in[f]$ (here [ ] denotes conjugacy class), we can decide whether $\alpha$ lies to the left, right, or on $f$.

Remark. The above argument shows that any pair $(f, \alpha)$ is good when $\alpha^{2}=$ id. When $G$ does not contain elements of order 2 , then it is more difficult to decide if an elliptic element lies to the left or right of an axis. The notion of on does not seem to make sense, (in an elementary way) for elliptic elements of odd order.

Step 2. Extend the action to $\stackrel{\circ}{D}^{2}$. 


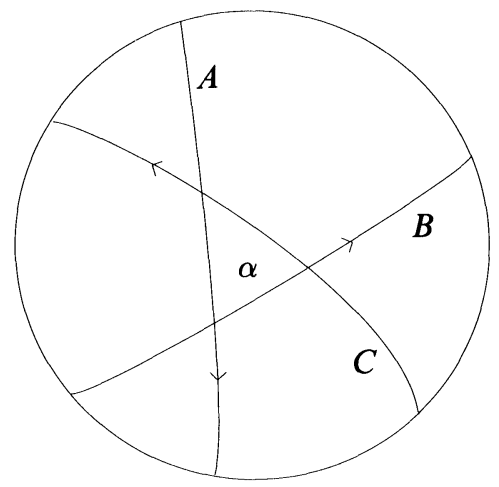

A Solution

Find an $\alpha$ to the left of each of $A, B$, and $C$

Figure 3

Remark. This involves two substeps. First we show how to represent the elements of $[f]$ by lines in $D^{2}$ and how to represent the elements of $[\alpha]$ by points in $D^{2}$ so that the geometric configuration of points and lines pairwise respect the abstract notion of left, right, and on. This makes the configuration of axes combinatorially more rigid. The geometric data allows us to put a metric space structure on the points corresponding to $[\alpha]$ where $d\left(\alpha, \alpha^{\prime}\right)=$ the minimal number of axes a line must cross to get from $\alpha$ to $\alpha^{\prime}$. It turns out that we can define a $G$ equivariant cell structure on $\stackrel{\circ}{D}^{2}$ whose vertices are essentially $[\alpha]$ and whose edges consist of shortest paths. By inspecting the action on the graph we see that $G$ is in fact a triangle group.

\section{ACKNOWLEDGMENT}

This research was initiated after Will Kazez pointed out to me that my idea of what the (classical) torus theorem said was incorrect. I would like to thank Bob Edwards, Paul Kirk, and Francis Bonahon for helpful conversations. 


\section{BIBLIOGRAPHY}

[E] D. B. A. Epstein, Periodic flows on 3-manifolds, Ann. of Math. (2) 95 (1972), 66-82.

[F1] C. D. Feustel, On the Torus theorem and its applications, Trans. Amer. Math. Soc. 217 (1976), 1-43.

[F2] - On the torus theorem for closed 3-manifolds, Trans. Amer. Math. Soc. 217 (1976), 45-57.

[G] D. Gabai, Convergence groups are Fuchsian groups, preprint.

[GH] C. Gordon and W. Heil, Cyclic normal subgroups of fundamental groups of 3-manifolds, Topology 14 (1975), 305-309.

[GM] F. W. Gehring and G. Martin, Discrete quasiconformal groups I, Proc. London Math. Soc. (3) 55 (1987), 331-358.

[J] K. Johannson, Homotopy equivalences of 3-manifolds with boundary, Lecture Notes in Math., vol. 761, Springer-Verlag, Berlin and New York, 1979.

[JS] W. H. Jaco and P. B. Shalen, Seifert fibered spaces in 3-manifolds, Mem. Amer. Math. Soc. 2 (1979).

[K] S. P. Kerckhoff, The Nielsen realization problem, Ann. of Math. (2) 117 (1983), 235-265.

[Kn] H. Kneser, Gerschlossene Flachen in dreidimensionalen Mannigfaltigkeiten, Jahres. der Deut. Math. Verein. 38 (1929), 248-260.

[M] G. Mess, The Seifert conjecture and groups which are coarse quasi isometric to planes, preprint.

[Mi] J. Milnor, A unique factorization theorem for 3-manifolds, Amer. J. Math. 84 (1962), 1-7.

[N] J. Nielsen, Abbildungsklassen endlicher Ordnung, Acta Math. 75 (1942), 23-115.

[S1] P. Scott, $A$ new proof of the annulus and torus theorems, Amer. J. Math. 2 (1978), 241-277.

[S2] _ There are no fake Seifert fibred spaces with infinite $\pi_{1}$, Ann. of Math. (2) 117 (1983), 35-70.

[Th] W. P. Thurston, Three dimensional manifolds, Kleinian groups, and hyperbolic geometry, Proc. Sympos. Pure Math. 39 (1983), 87-111.

[T] P. Tukia, Homeomorphic conjugates of Fuchsian groups, J. Reine. Agnew. Math. 391 (1988), 1-54.

[W] S. Wolpert, Geodesic length functions and the Nielson problem, J. Differential Geom. 25 (1987), 275-296.

[W1] F. Waldhausen, Gruppen mit zentrum und 3-dimensionale mannigfaltigkeiten, Topology 6 (1967), 505-517.

[W2] __, On the determination of some bounded 3-manifolds by their fundamental groups alone, Proc. Internat. Sympos. Topology, Hercy-Novi, Yugoslavia, 1968, pp. 331-332. 
[W3] - On irreducible 3-manifolds which are sufficiently large, Ann. of Math. (2) 87 (1968), 56-88.

[Z] H. Zieschang, Finite groups of mapping classes of surfaces, Lecture Notes in Math., vol. 875, Springer-Verlag, Berlin and New York, 1981.

Department of Mathematics, California Institute of Technology, Pasadena, California 91125

E-mail address: gabai@ juliet.caltech.edu 